Article

\title{
Garcinol Enhances TRAIL-Induced Apoptotic Cell Death through Up-Regulation of DR5 and Down-Regulation of c-FLIP Expression
}

\author{
Seok Kim ${ }^{1}$, Seung Un Seo ${ }^{1}$, Kyoung-Jin Min ${ }^{1}$, Seon Min Woo ${ }^{1}$, Ju-Ock Nam ${ }^{2}$, \\ Peter Kubatka $^{3,4}$, Shin Kim ${ }^{1}$ (iD , Jong-Wook Park ${ }^{1}$ and Taeg Kyu Kwon ${ }^{1, *}$ (iD \\ 1 Department of Immunology and School of Medicine, Keimyung University, 2800 Dalgubeoldaero, \\ Dalseo-Gu, Daegu 42601, Korea; kimseok216@naver.com (S.K.); ssu3885@gmail.com (S.U.S.); \\ Kyoungjin.min@gmail.com (K.-J.M.); woosm724@gmail.com (S.M.W.); god98005@dsmc.or.kr (S.K.); \\ j303nih@dsmc.or.kr (J.-W.P) \\ 2 Department of Food Science and Biotechnology, Kyungpook National University, Daegu 41566, Korea; \\ namjo73@gmail.com \\ 3 Department of Medical Biology, Jessenius Faculty of Medicine, Comenius University in Bratislava, \\ Martin 03601, Slovakia; kubatkap@gmail.com \\ 4 Department of Experimental Carcinogenesis, Division of Oncology, Biomedical Center Martin, \\ Jessenius Faculty of Medicine, Comenius University in Bratislava, Martin 03601, Slovakia \\ * Correspondence: kwontk@dsmc.or.kr; Tel.: +82-53-5803882; Fax: +82-53-5803795
}

Received: 11 June 2018; Accepted: 29 June 2018; Published: 2 July 2018

\begin{abstract}
Garcinol is a polyisoprenylated benzophenone derived from the Garcinia indica fruit that possess potential therapeutic effects such as inhibition of inflammation and tumor expansion. Here, we investigated whether garcinol induces TRAIL sensitization in renal carcinoma cells. Single treatment with garcinol or TRAIL did not effect on apoptosis. However, combined treatment with garcinol plus TRAIL significantly induced apoptosis in renal carcinoma (Caki, ACHN and A498), lung carcinoma (A549) an hepatoma (SK-Hep1) cells. In contrast, garcinol plus TRAIL did not alter cell viability in normal cells. Garcinol plus TRAIL induced up-regulation of DR5 and down-regulation of c-FLIP expression at post-translational levels. Furthermore, knock-down of DR5 by siRNA and ectopic expression of c-FLIP blocked apoptotic cell death induced by garcinol plus TRAIL. Overall, our study provides evidence that garcinol can be exploited as a potential TRAIL sensitizer.
\end{abstract}

Keywords: garcinol; TRAIL; c-FLIP; DR5; apoptosis

\section{Introduction}

Garcinol is a polyisoprenylated benzophenone which may be isolated from the dried rind of the fruit Garcinia indica. It exhibits multiple biological effects, such as anti-inflammatory, anti-microbial an anti-oxidative activities [1-3]. Garcinol induces apoptosis via interference of the multiple signaling pathways, such as inactivation of STAT-3, NF- $\mathrm{kB}$ an PI3K/Akt signaling pathways [4-6]. Garcinol also inhibits proliferation of tumor cells, angiogenesis an cell cycle progression an induces apoptosis via inhibition of NF- $\mathrm{kB}$ and cyclooxygenase-2 expression in oral cancer [7]. Furthermore, garcinol has a sensitizing effect in combined treatment with cisplatin or TRAIL [8,9]. However, the molecular mechanisms of this anti-cancer effect by garcinol are not well understood.

TRAIL selectively induces cell death in cancer cells [10,11]. However, a lot of cancer cells reveal resistance to TRAIL through multiple mechanisms, including down-regulation of death receptor (DR)4/5 an up-regulation of decoy death receptors and anti-apoptotic proteins [12-14]. 
New approaches to overcome TRAIL resistance that combined treatment with pharmacological agents that modify the function of tumor-dysregulated apoptotic genes have been investigated [15-17].

In this present study, we investigated the molecular mechanisms involved in the sensitizing effect of garcinol on TRAIL-induced apoptosis in cancer cells.

\section{Results}

\subsection{Effect of Garcinol on TRAIL Sensitization}

To investigate whether garcinol enhances TRAIL sensitization, we employed renal carcinoma Caki cells that are resistant to TRAIL. Cells were treated with garcinol alone $(1$ and $2 \mu \mathrm{M})$, TRAIL alone (50 ng/mL) an co-treatment with garcinol and TRAIL. We assayed amount of apoptotic cell death by sub-G1 population and PARP cleavage. Garcinol plus TRAIL increased the sub-G1 population and PARP cleavage (Figure 1A). However, single treatment with garcinol or TRAIL did not induce apoptosis. Garcinol plus TRAIL induced apoptotic morphologies, such as cell shrinkage, apoptotic body formation an cell detachment on the plate (Figure 1B), nuclear condensation (Figure 1C) an the DNA fragmentation (Figure 1D). Furthermore, combined treatment with garcinol plus TRAIL increased caspase-3 activity (Figure 1E). To investigate the role of caspase activation in the garcinol plus TRAIL-induced apoptosis, we used a pan-caspase inhibitor, z-VAD-fmk. As shown in Figure 1F, z-VAD-fmk inhibited combined treatment-induced sub-G1 population and cleavage of PARP and caspase-3. Next, to investigate the molecular mechanism underlying the Caki cell death via combined treatment with garcinol and TRAIL, we analyzed the expression levels of apoptosis related proteins. Garcinol markedly induced up-regulation of DR5 and down-regulation of c-FLIP. However, other apoptosis related proteins (XIAP, survivin, DR4, Mcl-1 an Bcl-2) were not changed (Figure 1G). Taken together, these data suggest that garcinol enhances TRAIL-induced apoptosis in renal carcinoma Caki cells.

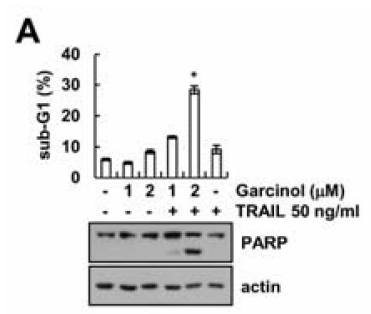

D

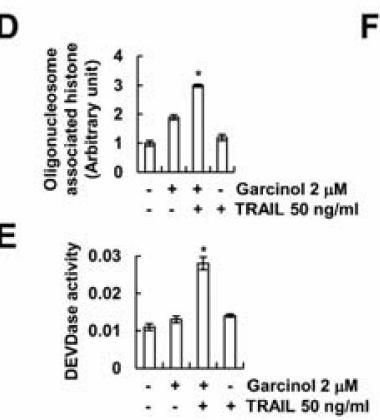

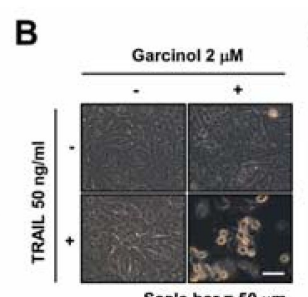

Scale bar $=50 \mu \mathrm{m}$

F

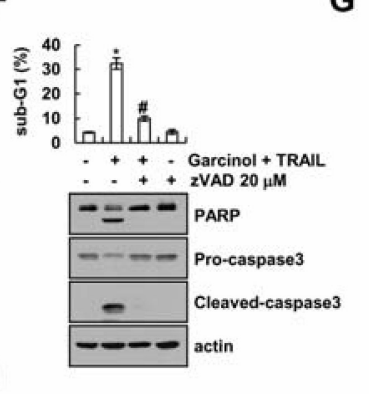

C

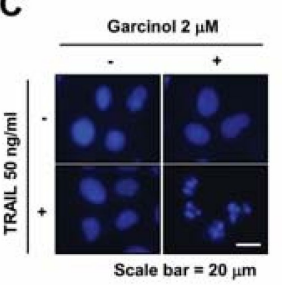

G

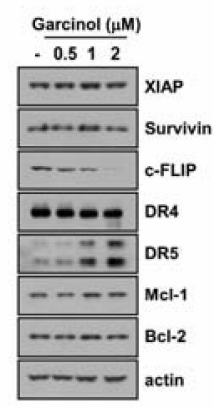

Figure 1. Garcinol sensitizes Caki cells to TRAIL-mediated apoptosis. (A-E) Caki cells were treated with garcinol $(1-2 \mu \mathrm{M})$ and/or $50 \mathrm{ng} / \mathrm{mL}$ TRAIL for $24 \mathrm{~h}$. Levels of apoptosis were assessed by flow cytometry an western blot showing the PARP and actin (A). Morphology of cells was visualized with an optical microscope (B). DAPI staining detected condensation and fragmentation of nuclei (C). Detection of DNA fragmentation (D) and caspase activity (E). (F) Caki cells were treated with $2 \mu \mathrm{M}$ garcinol and $50 \mathrm{ng} / \mathrm{mL}$ TRAIL for $24 \mathrm{~h}$ in the presence or absence of $20 \mu \mathrm{M} z$-VAD. Levels of apoptosis were assessed by flow cytometry an western blot showing the PARP, pro-caspase-3, cleaved caspase-3 and actin. (G) Caki cells were treated with $(0.5-2 \mu \mathrm{M})$ galcinol for $24 \mathrm{~h}$. The related levels of proteins were detected by western blot using indicated antibody. ${ }^{*} p<0.01$ compared to the control. $\# p<0.01$ compared to the garcinol plus TRAIL. 


\subsection{Garcinol Induces TRAIL Sensitization through Down-Regulation of c-FLIP Expression}

To investigate the role of c-FLIP protein in garcinol plus TRAIL-induced apoptosis, we examined mRNA and protein levels of c-FLIP in garcinol-treated cells. The mRNA levels of c-FLIP were not changed, but protein levels decreased in a time-dependent manner (Figure 2A). Next, we examined c-FLIP protein stability by garcinol treatment. The c-FLIP protein levels rapidly decreased in presence of cycloheximide (CHX) an were significantly lower in garcinol-treated cells than in vehicle-treated cells (Figure 2B). Pretreatment with proteasome inhibitors (MG132 and lactacystin) rescued the garcinol-mediated decrease of c-FLIP protein levels (Figure 2C) an inhibited induction of sub-G1 population and cleavage of PARP by combined treatment (Figure 2D). Therefore, our data suggested that garcinol decreases c-FLIP expression at post-translational levels in a proteasome-dependent pathway. Furthermore, ectopic expression of c-FLIP significantly inhibited apoptosis in garcinol plus TRAIL-treated cells (Figure 2E).

A

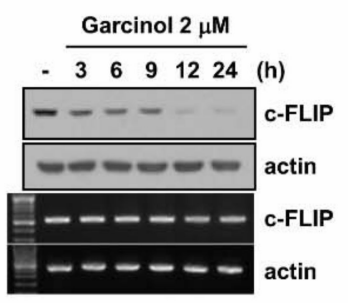

C

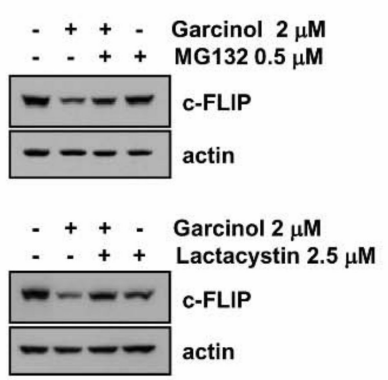

B
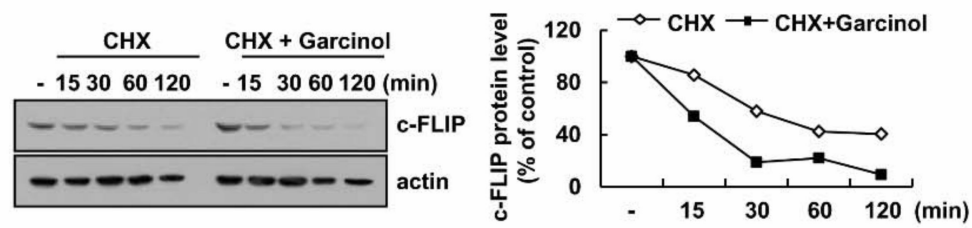

D

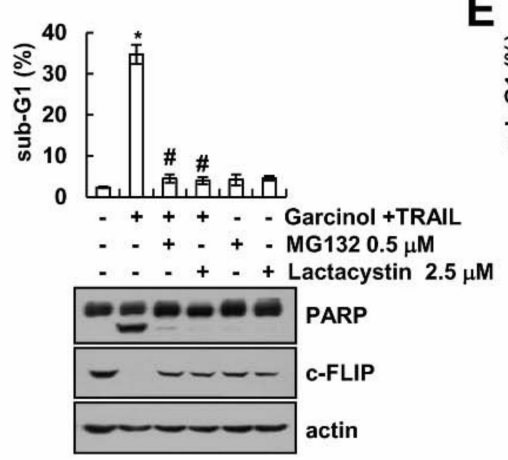

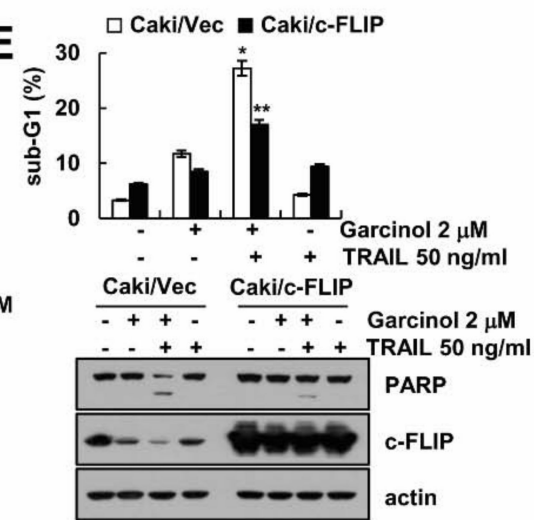

Figure 2. The effect of down-regulation of c-FLIP by garcinol treatment on TRAIL sensitization. (A) Caki cells were treated with $2 \mu \mathrm{M}$ garcinol for 3-24 h. Western blot (upper) and RT-PCR (lower) showing the c-FLIP and actin; (B) Caki cells were treated with $20 \mu \mathrm{g} / \mathrm{mL}$ cycloheximide (CHX) in the presence or absence of $2 \mu \mathrm{M}$ garcinol for 15-120 min. Western blot showing the c-FLIP and actin. The band intensity was calculated using Image J; (C) After pretreatment of $0.5 \mu \mathrm{M}$ MG132 and $2.5 \mu \mathrm{M}$ lactacystin for $30 \mathrm{~min}$, Caki cells were treated with $2 \mu \mathrm{M}$ garcinol for $24 \mathrm{~h}$. Western blot showing the c-FLIP and actin; (D) After pretreatment of $0.5 \mu \mathrm{M}$ MG132 and $2.5 \mu \mathrm{M}$ lactacystin for $30 \mathrm{~min}$, Caki cells were treated with $2 \mu \mathrm{M}$ garcinol and $50 \mathrm{ng} / \mathrm{mL}$ TRAIL for $24 \mathrm{~h}$. Levels of apoptosis were assessed by flow cytometry an western blot showing the PARP, c-FLIP and actin; (E) Vector cells (Caki/Vec) and c-FLIP-overexpressed cells (Caki/c-FLIP) were treated with $2 \mu \mathrm{M}$ garcinol and/or $50 \mathrm{ng} / \mathrm{mL}$ for $24 \mathrm{~h}$. Levels of apoptosis were assessed by flow cytometry an western blot showing the PARP, c-FLIP and actin. ${ }^{*} p<0.01$ compared to the control. \# $p<0.01$ compared to the garcinol plus TRAIL. ${ }^{* *} p<0.01$ compared to the garcinol plus TRAIL-treated Caki/Vec.

\subsection{Combined Treatment Garcinol and TRAIL Induces Up-Regulation of DR5 Expression}

Garcinol induced up-regulation of DR5 protein levels, but mRNA levels of DR5 were not alterd (Figure 3A). Garcinol markedly enhanced DR5 protein stability (Figure 3B). Next, we investigated whether up-regulation of proteasome subunits was involved in garcinol-induced DR5 up-regulation. 
The expression levels of two critical proteasome subunits (PSMA5 and PSMD4/S5a) were not changed by garcinol treatment (Figure 3C). In addition, expression of E3 ligases (Itch and Cbl) for DR5 also did not change in garcinol-treated cells (Figure 3C). Therefore, we need further study to identify the E3 ubiquitin ligase or deubiquitinase involved in garcinol-induced DR5 protein up-regulation. Garcinol also increased surface expression levels of DR5 (Figure 3D). To investigate the role of DR5 up-regulation in garcinol plus TRAIL-induced apoptosis, cells were transfected with DR5 siRNA. Knock-down of DR5 expression by siRNA significantly inhibited apoptosis (Figure 3E).

A

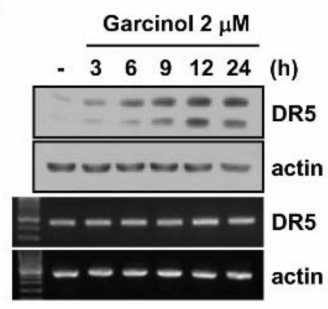

C

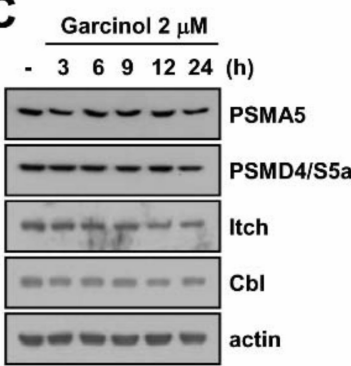

B
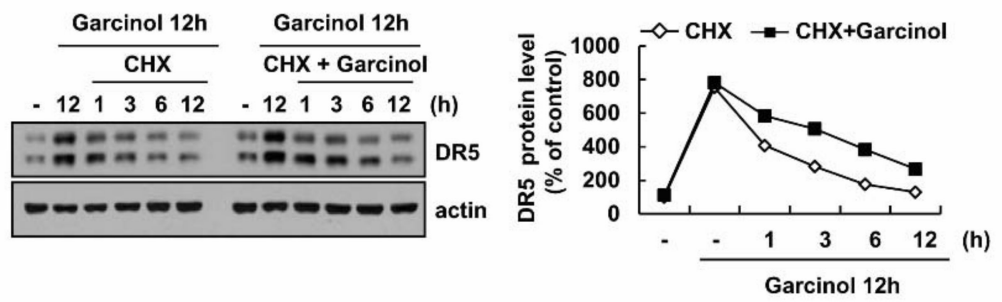

E

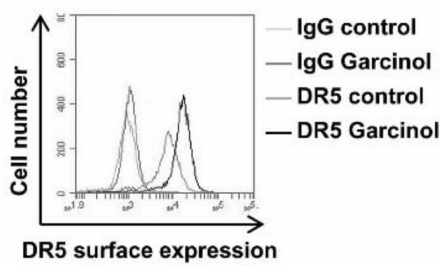

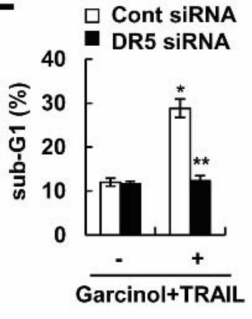

Cont DR5 SiRNA SiRNA ++ Garcinol+TRAIL $-0--1$

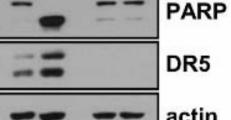

$-\infty$ actin

Figure 3. The effect of up-regulation of DR5 by garcinol on TRAIL sensitization. (A) Caki cells were treated with $2 \mu \mathrm{M}$ of garcinol for 3-24 h. Western blot (upper) and RT-PCR (lower) showing the DR5 and actin; (B) After garcinol treatment for $12 \mathrm{~h}$, Caki cells were treated with $20 \mu \mathrm{g} / \mathrm{mL}$ cycloheximide $(\mathrm{CHX})$ in the presence or absence of $2 \mu \mathrm{M}$ garcinol for 1-12 $\mathrm{h}$. Western blot showing the DR5 and actin. The band intensity was calculated using Image J; (C) Caki cells were treated with $2 \mu \mathrm{M}$ garcinol for 3-24 h. Western blot showing the PSMA5, PSMD4/S5a, Itch, Cbl and actin; (D) For DR5 surface staining, cells were treated with $2 \mu \mathrm{M}$ garcinol for $24 \mathrm{~h}$ and the cell surface expression level of DR5 was determined by flow cytometry as mentioned in a material and methods; (E) Caki cells were transfected with control or DR5 siRNA an then cells were treated with $2 \mu \mathrm{M}$ garcinol plus $50 \mathrm{ng} / \mathrm{mL}$ TRAIL for $24 \mathrm{~h}$. Levels of apoptosis were assessed by flow cytometry an western blot showing the PARP, DR5 and actin. ${ }^{*} p<0.01$ compared to the control. ${ }^{* *} p<0.01$ compared to the garcinol plus TRAIL-treated control siRNA.

\subsection{Garcinol-Mediated TRAIL Sensitization Is Not Associated with Reactive Oxygen Species (ROS) Signaling Pathway}

Generation of ROS is linked to the TRAIL-mediated apoptosis [18,19]. Garcinol induced generation of ROS which was analyzed by $\mathrm{H}_{2}$ DCF-DA-based fluorescence microscopy and FACS (Figure 4A). Therefore, we examined whether ROS are involved in TRAIL sensitization by garcinol treatment. ROS scavengers (NAC, GEE and Trolox) did not abolish garcinol plus TRAIL-induced apoptosis and cleavage of PARP (Figure 4B) an modulation of DR5 and c-FLIP expression (Figure 4B). Therefore, garcinol-induced TRAIL sensitization is independent of ROS signaling. 
A
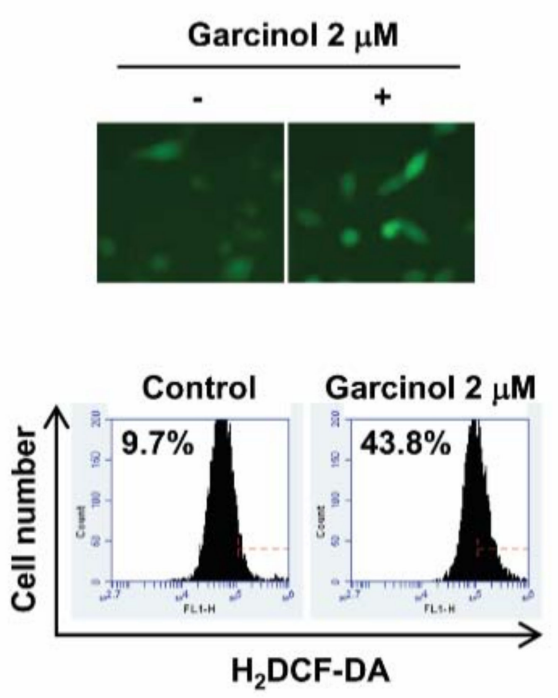

B

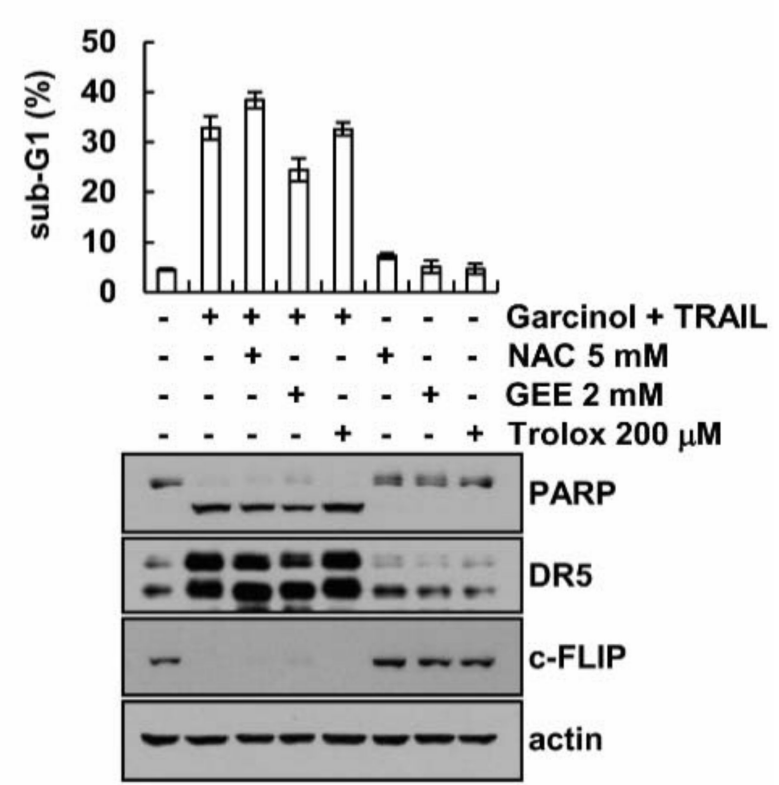

Figure 4. TRAIL sensitization by garcinol is independent of ROS levels. (A) For measurement of ROS production, cells were treated with $2 \mu \mathrm{M}$ garcinol for $2 \mathrm{~h}$ and then stained with $\mathrm{H}_{2}$ DCF-DA dye. Fluorescence was detected by fluorescence microscopy and flow cytometry; (B) After pretreatment with the indicated concentrations of NAC, GEE and trolox for $30 \mathrm{~min}$, cells were stimulated with $2 \mu \mathrm{M}$ garcinol plus $50 \mathrm{ng} / \mathrm{mL}$ TRAIL for $24 \mathrm{~h}$. Levels of apoptosis were assessed by flow cytometry an western blot showing the PARP, DR5, c-FLIP and actin.

2.5. Garcinol Induces TRAIL Sensitization in Other Cancer Cells, but Garcinol Plus TRAIL Had No Effect on Apoptosis in Normal Cells

Next, we investigated whether garcinol also induces TRAIL sensitization in other types of cancer cells and normal cells. Garcinol induced TRAIL sensitization in other renal carcinoma (ACHN and A498), lung carcinoma (A549) an hepatoma (SK-Hep1) cells (Figure 5A). Expression patterns of DR5 and c-FLIP in other cancer cells are similar to that of Caki cells (Figure 5B). However, garcinol did not alter the sensitivity against TRAIL treatment in normal human mesangial cells (MC) and normal mouse renal tubular epithelial (TCMK-1) (Figure 5C). These data indicate that garcinol enhances TRAIL-mediated apoptotic cell death in cancer cells. 
A

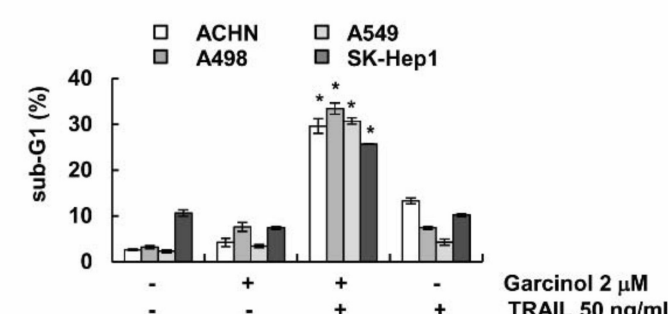

-++ - Garcinol $2 \mu \mathrm{M}$

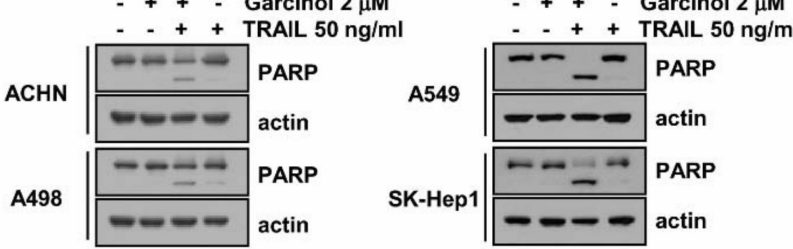

C

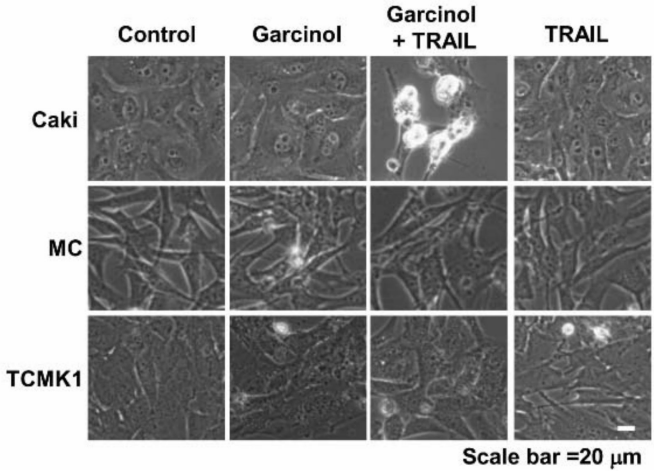

B $\quad \begin{array}{rll}-0.512 & 2 & \text { Garcinol }(\mu \mathrm{M})\end{array}$

ACHN

A498

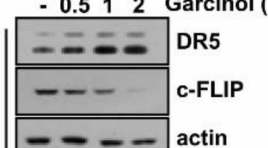

A549
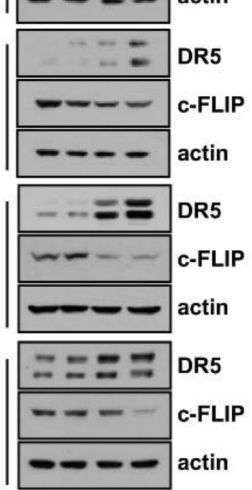

SK-Hep1 actin

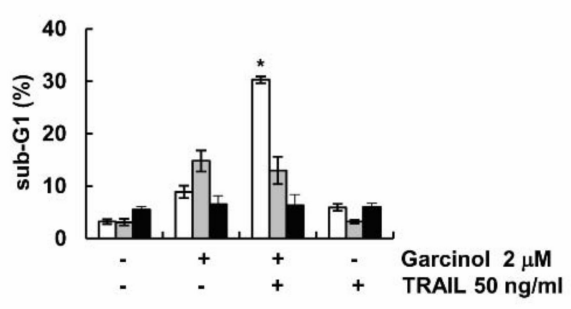

Figure 5. The effects of garcinol on TRAIL sensitization in cancer and normal cells. (A) ACHN, A498 (renal carcinoma), A549 (lung carcinoma) and SK-Hep1 (liver carcinoma) cells were treated with $2 \mu \mathrm{M}$ garcinol and/or $50 \mathrm{ng} / \mathrm{mL}$ TRAIL for $24 \mathrm{~h}$. Levels of apoptosis were assessed by flow cytometry an western blot showing the PARP and actin; (B) Cells were treated with $0.5-2 \mu \mathrm{M}$ garcinol for $24 \mathrm{~h}$. The related levels of proteins were detected by western blot using indicated antibody; (C) Caki, masangial cells (MC) and TCMK-1 cells were treated with $2 \mu \mathrm{M}$ garcinol and/or $50 \mathrm{ng} / \mathrm{mL}$ TRAIL for $24 \mathrm{~h}$. Cell morphology was explored using an interference optical microscope. Levels of apoptosis were assessed by flow cytometry. ${ }^{*} p<0.01$ compared to the control.

\section{Discussion}

Here, we found that garcinol enhanced TRAIL sensitization in renal carcinoma cells an induced up-regulation of DR5 and down-regulation of c-FLIP. Combined treatment with garcinol plus TRAIL induced apoptotic cell death in cancer cells, but not in normal cells. These results suggest that garcinol could be an attractive TRAIL sensitizer.

Garcinol induced apoptosis in several cancer cell lines through multiple signaling pathways. The apoptotic effects of garcinol were correlated with inactivation of STAT-3, NF- $\mathrm{KB}$ an PI3K/Akt signaling pathways [4-6]. However, whether garcinol exhibits similar activity in renal carcinoma cell lines has not yet been evaluated. Garcinol revealed cytotoxicity with $\mathrm{IC}_{50}$ of $10 \sim 20 \mu \mathrm{M}$ in colorectal cancer cell line HT-29 and breast cancer cell line MCF-7, respectively [5,6]. In our study, low concentration of garcinol $(2 \mu \mathrm{M})$ did not induce apoptotic cell death at $24 \mathrm{~h}$ in Caki cells. However, combined treatment garcinol $(1-2 \mu \mathrm{M})$ and TRAIL caused apoptotic cell death in Caki, ACHN, A498, A549 an SK-Hep1 cells, but not normal cells. Prasad et al., also reported that garcinol potentiated TRAIL-induced apoptosis through modulation of death receptors and anti-apoptotic proteins [9]. Interestingly, they reported that the sensitizing effect of garcinol was mediated through ROS generation in HCT116 cells. However, ROS scavengers (NAC, GEE and trolox) did not inhibit combined treatment with garcinol plus TRAIL-induced apoptosis in 
our cell culture conditions (Figure 4B). We assumed that this discrepancy was caused by different cell types or experimental conditions. Prasad's data showed that inhibitory effect of ROS scavengers only detected at high concentration of $10 \mathrm{mM}$ NAC and $10 \mathrm{mM} \mathrm{GSH} \mathrm{[9].} \mathrm{High} \mathrm{concentration} \mathrm{of}$ ROS scavengers induced unexpected results. In addition, we used a low concentration of garcinol $(2 \mu \mathrm{M})$, which did not induce cell death. However, they used high concentration of garcinol $(15 \mu \mathrm{M})$. High concentrations of garcinol generate more ROS than our systems. Thus, relative amounts of ROS result in different effects on cell death. Furthermore, multiple studies suggested that down-regulation of c-FLIP may play a critical role in TRAIL-induced apoptosis. Recently, our group reported that cathepsin S inhibitor induced down-regulation of c-FLIP expression through up-regulation of $\mathrm{Cbl}$ expression [20]. Cathepsin S inhibitor-induced mitochondrial ROS generation played a critical role in c-FLIP down-regulation [20]. ROS play important roles in post-translational regulation of c-FLIP protein through multiple mechanisms $[18,19]$. Therefore, we need further experiments to identify the ROS-dependent or -independent mechanisms involved in garcinol-induced modulation of apoptosis-related proteins.

In addition, we demonstrated that combined treatment with garcinol plus TRAIL induced up-regulation of DR5 and down-regulation of c-FLIP expression through modulation of protein stability. Garcinol did not affect Itch and Cbl E3 ligase expression. Recently, Oh et al., reported that knock-down of monocyte chemotactic protein-induced protein-1 (MCPIP1) suppresses DR5 deubiquitination [21]. However, we could not rule out the possibility of involvement of other E3 ligase and de-ubiquitinases. There are clearly different from the effects of garcinol on protein stability in DR5 and c-FLIP expression. Therefore, which E3 ligases or de-ubiquitinases are involved in the regulation of DR5 an c-FLIP requires further study. Taken together, our data showed that garcinol sensitized TRAIL-induced apoptosis in various cancer cells, but not in normal cells. Therefore, our data suggest that garcinol might be an attractive sensitizer for TRAIL resistance cancer cells.

\section{Materials and Methods}

\subsection{Cell Cultures and Materials}

Mouse kidney cells (TCMK-1) were a gift from Dr. T.J. Lee (Yeungnam University, Korea). Primary culture of human mesangial cells (Cryo NHMC) were purchased from Clonetics (San Diego, CA, USA) an other cell lines are from the ATCC (Manassas, VA, USA). All cells were maintained in Dulbecco's modified Eagle's medium an supplemented with $10 \%$ FBS, 5\% antibiotic solution an $100 \mu \mathrm{g} / \mathrm{mL}$ gentamycin. Garcinol and lactacystin was purchased from Enzo Life Sciences (Ann Arbor, MI, USA). Recombinant human TRAIL and z-VAD-fmk were purchased from R\&D (Minneapolis, MN, USA). Calbiochem supplied N-acetyl-L-cysteine (NAC), trolox an MG132 (San Diego, CA, USA). The following antibodies were used: anti-XIAP, -Bcl-2, -Mcl-1, -survivin, -Cbl an -Itch (Santa Cruz Biotechnology, Santa Cruz, CA, USA); anti-c-FLIP (ALEXIS Corporation, San Diego, CA, USA); anti-PARP, -DR5, -PSMA5, -PSMD4/S5a, -cleaved caspase-3 (Cell Signaling Technology, Beverly, MA, USA); anti-caspase-3 (Enzo Life Sciences, Ann Arbor, MI, USA); anti-DR4 (Abcam, Cambridge, MA, USA); anti-actin (Sigma-Aldrich, St. Louis, MO, USA). Santa Cruz Biotechnology (Santa Cruz, CA, USA) and Bioneer (Daejeon, Korea) supplied DR5 siRNA and GFP siRNA (control siRNA), respectively. Sigma Chemical Co. supplied other reagents (St. Louis, MO, USA).

\subsection{Flow Cytometry and Western Blot Analysis}

We performed the western blot using the RIPA lysis buffer [22,23] and flow cytometry analysis as described in our study [20].

\subsection{4',6'-Diamidino-2-Phenylindole Staining (DAPI) Staining and DNA Fragmentation Assay}

For detection of DAPI staining and DNA fragmentation, we used $300 \mathrm{nM}$ DAPI solution (Roche, Mannheim, Germany) an the cell death detection ELISA plus kit (Boehringer Mannheim, Indianapolis, IN, USA) [20]. 


\subsection{Detection of Caspase-3 Activity}

To evaluate caspase- 3 activity, cell lysates were obtained in $100 \mu \mathrm{L}$ of reaction buffer and then measured activities with a spectrophotometer at $405 \mathrm{~nm}$ [20].

\subsection{Reverse Transcription-Polymerase Chain Reaction (RT-PCR)}

To obtain cDNA, total RNA was prepared using the TriZol reagent (Life Technologies; Gaithersburg, MD, USA) an used M-MLV reverse transcriptase (Gibco-BRL; Gaithersburg, MD, USA). For PCR, we used DNA polymerase with primers targeting actin , c-FLIP an DR5 [20].

\subsection{Detection of DR5 on Cell Surface}

Detached cells by $0.2 \%$ EDTA were washed with PBS an then suspended in $100 \mu \mathrm{M}$ PBS including 10\% FCS and 1\% sodium azide an added to the primary antibody (DR5-phycoerythrin; Abcam, Cambridge, MA, USA) for $1 \mathrm{~h}$ at room temperature. Then, the cells washed with PBS including $10 \%$ FCS and 1\% sodium azide an suspended in $400 \mu \mathrm{L}$ of PBS for the detection of DR5 on cell surface by flow cytometry.

\subsection{Detection of Reactive Oxygen Species (ROS) Production}

We detected levels of ROS production using $2^{\prime}, 7^{\prime}$-dichlorodihydrofluorescein diacetate $\left(\mathrm{H}_{2}\right.$ DCF-DA). Cells were incubated with $10 \mu \mathrm{M} \mathrm{H} \mathrm{H}_{2} \mathrm{DCF}-\mathrm{DA}$ for $10 \mathrm{~min}$ an then fluorescence was detected.

\subsection{Statistical Analysis}

The values in all graphs represent the mean \pm SD from three independent samples. The data were analyzed using a one-way ANOVA and post-hoc comparisons (Student-Newman-Keuls) using the SPSS 22.0 software (SPSS Inc., Chicago, IL, USA).

Author Contributions: S.K., J.-W.P. and T.K.K. conceived and designed the experiments; S.K., S.M.W. and S.U.S. performed the experiments; S.K., K.-J. M., J.-O.N., P.K., S.K., J.-W.P. and T.K.K. analyzed the data; T.K.K. contributed reagents/materials/analysis tools; S.K., K.-J.M., S.U.S. and T.K.K. wrote the paper.

Funding: This work was supported by an NRF grant funded by the Korea Government (MSIP) (2014R1A5A2010008 and NRF-2016R1A2B2013393) and a 2018 Scholar Research Grant from Keimyung University.

Conflicts of Interest: The authors declare that they have no conflict of interest.

\section{Abbreviations}

TRAIL Tumor necrosis factor-related apoptosis-inducing ligand

STAT Signal transducer and activator of transcription

NF- $\kappa$ B Nuclear factor- $\kappa B$

PI3K Phosphatidylinositol-4,5-bisphosphate 3-kinase

ROS Reactive oxygen species

DR Death receptor

cFLIP Cellular FADD-like IL-1 $\beta$-converting enzyme-inhibitory protein

CHX cycloheximide

PSMA5 20S proteasome subunit type 5

PSMD4/S5a 26S proteasome non-ATPase regulatory 4 


\section{References}

1. Yamaguchi, F.; Ariga, T.; Yoshimura, Y.; Nakazawa, H. Antioxidative and anti-glycation activity of garcinol from Garcinia indica fruit rind. J. Agric. Food Chem. 2000, 48, 180-185. [CrossRef] [PubMed]

2. Chatterjee, A.; Yasmin, T.; Bagchi, D.; Stohs, S.J. The bactericidal effects of lactobacillus acidophilus, garcinol and protykin compared to clarithromycin, on helicobacter pylori. Mol. Cell. Biochem. 2003, 243, $29-35$. [CrossRef] [PubMed]

3. Liao, C.H.; Sang, S.; Liang, Y.C.; Ho, C.T.; Lin, J.K. Suppression of inducible nitric oxide synthase and cyclooxygenase-2 in downregulating nuclear factor-kappa b pathway by garcinol. Mol. Carcinog. 2004, 41, 140-149. [CrossRef] [PubMed]

4. Sethi, G.; Chatterjee, S.; Rajendran, P.; Li, F.; Shanmugam, M.K.; Wong, K.F.; Kumar, A.P.; Senapati, P.; Behera, A.K.; Hui, K.M.; et al. Inhibition of stat3 dimerization and acetylation by garcinol suppresses the growth of human hepatocellular carcinoma in vitro and in vivo. Mol. Cancer 2014, 13, 66. [CrossRef] [PubMed]

5. Liao, C.H.; Sang, S.; Ho, C.T.; Lin, J.K. Garcinol modulates tyrosine phosphorylation of FAK and subsequently induces apoptosis through down-regulation of Src, ERK an Akt survival signaling in human colon cancer cells. J. Cell. Biochem. 2005, 96, 155-169. [CrossRef] [PubMed]

6. Ahmad, A.; Wang, Z.; Ali, R.; Maitah, M.Y.; Kong, D.; Banerjee, S.; Padhye, S.; Sarkar, F.H. Apoptosis-inducing effect of garcinol is mediated by NF-кB signaling in breast cancer cells. J. Cell. Biochem. 2010, 109, 1134-1141. [CrossRef] [PubMed]

7. Aggarwal, S.; Das, S.N. Garcinol inhibits tumour cell proliferation, angiogenesis, cell cycle progression and induces apoptosis via NF-kB inhibition in oral cancer. Tumor Biol. 2016, 37, 7175-7184. [CrossRef] [PubMed]

8. Li, F.; Shanmugam, M.K.; Siveen, K.S.; Wang, F.; Ong, T.H.; Loo, S.Y.; Swamy, M.M.; Mandal, S.; Kumar, A.P.; Goh, B.C.; et al. Garcinol sensitizes human head and neck carcinoma to cisplatin in a xenograft mouse model despite downregulation of proliferative biomarkers. Oncotarget 2015, 6, 5147-5163. [CrossRef] [PubMed]

9. Prasad, S.; Ravindran, J.; Sung, B.; Pandey, M.K.; Aggarwal, B.B. Garcinol potentiates trail-induced apoptosis through modulation of death receptors and antiapoptotic proteins. Mol. Cancer Ther. 2010, 9, 856-868. [CrossRef] [PubMed]

10. Wiley, S.R.; Schooley, K.; Smolak, P.J.; Din, W.S.; Huang, C.P.; Nicholl, J.K.; Sutherland, G.R.; Smith, T.D.; Rauch, C.; Smith, C.A.; et al. Identification and characterization of a new member of the TNF family that induces apoptosis. Immunity 1995, 3, 673-682. [CrossRef]

11. Pitti, R.M.; Marsters, S.A.; Ruppert, S.; Donahue, C.J.; Moore, A.; Ashkenazi, A. Induction of apoptosis by APO-2 ligand, a new member of the tumor necrosis factor cytokine family. J. Biol. Chem. 1996, 271, 12687-12690. [CrossRef] [PubMed]

12. Eggert, A.; Grotzer, M.A.; Zuzak, T.J.; Wiewrodt, B.R.; Ho, R.; Ikegaki, N.; Brodeur, G.M. Resistance to tumor necrosis factor-related apoptosis-inducing ligand (trail)-induced apoptosis in neuroblastoma cells correlates with a loss of caspase-8 expression. Cancer Res. 2001, 61, 1314-1319. [PubMed]

13. Limami, Y.; Pinon, A.; Riaz, A.; Simon, A. Trail and targeting cancer cells: Between promises and obstacles. Cell. Mol. Biol. 2015, 61, 33-38. [PubMed]

14. O'Leary, L.; van der Sloot, A.M.; Reis, C.R.; Deegan, S.; Ryan, A.E.; Dhami, S.P.; Murillo, L.S.; Cool, R.H.; Correa de Sampaio, P.; Thompson, K.; et al. Decoy receptors block trail sensitivity at a supracellular level: The role of stromal cells in controlling tumour trail sensitivity. Oncogene 2016, 35, 1261-1270.

15. Han, M.A.; Lee, D.H.; Woo, S.M.; Seo, B.R.; Min, K.J.; Kim, S.; Park, J.W.; Kim, S.H.; Choi, Y.H.; Kwon, T.K. Galangin sensitizes trail-induced apoptosis through down-regulation of anti-apoptotic proteins in renal carcinoma CAKI cells. Sci. Rep. 2016, 6, 18642. [CrossRef] [PubMed]

16. Min, K.J.; Seo, B.R.; Bae, Y.C.; Yoo, Y.H.; Kwon, T.K. Antipsychotic agent thioridazine sensitizes renal carcinoma Caki cells to TRAIL-induced apoptosis through reactive oxygen species-mediated inhibition of AKT signaling and downregulation of Mcl-1 and c-FLIP(L). Cell Death Dis. 2014, 5, e1063. [CrossRef] [PubMed]

17. Trivedi, R.; Mishra, D.P. Trailing trail resistance: Novel targets for trail sensitization in cancer cells. Front. Oncol. 2015, 5, 69. [CrossRef] [PubMed] 
18. Wilkie-Grantham, R.P.; Matsuzawa, S.; Reed, J.C. Novel phosphorylation and ubiquitination sites regulate reactive oxygen species-dependent degradation of anti-apoptotic c-flip protein. J. Biol. Chem. 2013, 288, 12777-12790. [CrossRef] [PubMed]

19. Chanvorachote, P.; Nimmannit, U.; Wang, L.; Stehlik, C.; Lu, B.; Azad, N.; Rojanasakul, Y. Nitric oxide negatively regulates Fas CD95-induced apoptosis through inhibition of ubiquitin-proteasome-mediated degradation of FLICE inhibitory protein. J. Biol. Chem. 2005, 280, 42044-42050. [CrossRef] [PubMed]

20. Seo, B.R.; Min, K.J.; Woo, S.M.; Choe, M.; Choi, K.S.; Lee, Y.K.; Yoon, G.; Kwon, T.K. Inhibition of cathepsin $\mathrm{S}$ induces mitochondrial ROS that sensitizes TRAIL-mediated apoptosis through p53-mediated downregulation of Bcl-2 and c-FLIP. Antioxid. Redox Signal. 2017, 27, 215-233. [CrossRef] [PubMed]

21. Oh, Y.T.; Qian, G.; Deng, J.; Sun, S.Y. Monocyte chemotactic protein-induced protein-1 enhances DR5 degradation and negatively regulates dr5 activation-induced apoptosis through its deubiquitinase function. Oncogene 2018, 37, 3415-3425. [CrossRef] [PubMed]

22. Park, Y.S.; Kwon, Y.J.; Chun, Y.J. CYP1B1 activates Wnt/ $\beta$-catenin signaling through suppression of HERC5-mediated ISGylation for protein degradation on $\beta$-catenin in HeLa cells. Toxicol. Res. 2017, 33, 211-218. [CrossRef] [PubMed]

23. Jo, Y.; Shin, D.Y. Repression of the F-box protein Skp2 is essential for actin damage-induced tetraploid G1 arrest. BMB Rep. 2017, 50, 379-383. [CrossRef] [PubMed]

Sample Availability: Samples of the compounds are available from the authors. 